\title{
Biosynthetic and environmental effects on the stable carbon isotopic compositions of anteiso - (3-methyl) and iso- (2-methyl) alkanes in tobacco leaves
}

\begin{abstract}
Nicotiana tabacum is the only plant known to synthesise large quantities of anteiso- (3methyl) alkanesand iso- (2-methyl) alkanes. We investigated the carbon isotope ratios of individual long-chain n-alkanes, anteiso- and iso-alkanes (in the $\mathrm{C}_{23}-\mathrm{C}_{33}$ carbon number range) extracted from tobacco grown in chambers under controlled conditions to confirm the pathway used by the tobacco plant to synthesise these particular lipids and to examine whether environmental data are recorded in these compounds. Tobacco was grown under differing temperatures, water availabilities and light intensities in order to control its stable carbon isotope ratios and evaluate isotopic fractionations associated with the synthesis of these particular lipids. The anteiso-alkanes were found to have a predominant even-carbon number distribution (maximising at $\mathrm{C}_{32}$ ), whereas the isoalkanes exhibit an odd-carbon number distribution (maximising at $\mathrm{C}_{31}$ ). Iso-alkanes were relatively more abundant than the anteiso-alkanes and only two anteiso-alkanes $\left(\mathrm{C}_{30}\right.$ and $\mathrm{C}_{32}$ ) were observed.
\end{abstract}

The anteiso-alkanes and iso-alkanes were found to be enriched in ${ }^{13} \mathrm{C}$ by $2.8-4.3 \%$ and 0 $1.8 \%$ compared to the n-alkanes, respectively, consistent with different biosynthetic precursors. The assumed precursor for the odd-carbon-numbered iso-alkanes is isobutyryl-CoA (a $\mathrm{C}_{4}$ unit derived from valine) followed by subsequent elongation of $\mathrm{C}_{2}$ units and then decarboxylation. The assumed precursor for even-carbon-numbered anteiso-alkanes is a-methylbutyryl-CoA (a $\mathrm{C}_{5}$ unit derived from isoleucine) and subsequent elongation by $\mathrm{C}_{2}$ units followed by decarboxylation. The ratio of carbon atoms derived from a-methylbutyryl-CoA and subsequent $\mathrm{C}_{2}$ units (from malonyl-CoA) is 1:5 for the biosynthesis of a $\mathrm{C}_{30}$ anteiso-alkane. The ratio of carbon atoms derived from iso-butyryl-CoA and subsequent $\mathrm{C}_{2}$ units (from malonyl-CoA) is 4:25 for the synthesis of a $\mathrm{C}_{29}$ iso-alkane. An order of ${ }^{13} \mathrm{C}$ depletion $n$-alkanes $>$ iso-alkanes $>$ anteiso-alkanes is evident from compound specific isotope data. This trend can probably be attributed to the ratio of the two different sources of carbon atoms in the final wax components.

Higher water availability generally results in more depleted stable carbon isotope ratios due to maximised discrimination during carboxylation, associated with less diffusional limitation. This was confirmed in the present study by compound specific isotope analyses of iso-alkanes, anteiso-alkanes and n-alkane lipids extracted from the tobacco leaves. Likewise, light intensity has been shown to influence plant bulk $\delta^{13} \mathrm{C}$ in previous studies. The carbon isotope ratios of n-alkanes in tobacco grown under lowlight conditions were about $2 \%$ more depleted in ${ }^{13} \mathrm{C}$ than those of lipids extracted from tobacco grown under elevated light conditions. A similar order of difference is observed for the iso-alkanes and anteiso-alkanes (1.8\%o and 1.9\%o, respectively). A negligible depletion in carbon isotope ratios was observed for the iso-alkanes and anteiso-alkanes 
extracted from tobacco grown under elevated temperatures. These results are consistent with the work of Farquhar [Farquhar, G.D., 1980. Carbon isotope discrimination by plants: effects of carbon dioxide concentration and temperature via the ratio of intercellular and atmospheric $\mathrm{CO}_{2}$ concentrations. In: Pearman, G.I. (Ed.), Carbon Dioxide and Climate: Australian Research. Springer, Berlin, pp. 105-110] where temperature appears to have only a minor effect on plant bulk $\delta^{13} \mathrm{C}$. 